\title{
Developmental Screening Tools for Motor Developmental Delay in High Risk Preterm Infants
}

\author{
Ramachandran $\mathbf{S}^{1}$, Dutta $\mathbf{S}^{2}$
}

\begin{abstract}
The preterm very low birth weight infants are at high risk of motor developmental delays. The developmental screening tools used by health professionals in pediatric practice serves several purposes viz. as a discriminative screening tool to identify the developmental delay in such high risk infants; as an evaluation tool to quantify the levels of functional skills achieved; as a prognostic tool to quantify the changes in levels of functional skills following specific developmental care interventions and as a predictive tool to predict the quantum of existing or impending neuro-developmental disability in high risk infants. Thus developmental screening tools serves as an integral part of early intervention programs. Such screening tools also serve as program evaluation strategy in quantifying the efficacy of early developmental care intervention programs. This review is aimed at describing the properties of developmental screening tools for motor developmental delay in preterm infants.
\end{abstract}

Key words: Developmental Screening, Early Intervention, Clinimetric, Psychometric, Preterm, High-risk

\section{Introduction}

\section{Need for standardized developmental screening tool}

$T$ he adverse neurodevelopmental outcomes are often associated with prematurity ${ }^{1,2}$. The preterm infants are nursed in Neonatal Intensive Care Units (NICU) as there is an invariable difficulty in coping up with environmental demands owing to its early exposure to the environment. The survival rates of such preterm infants have increased with due NICU care; on the contrary these infants presents with neuro-developmental disorder at later stages of which motor developmental delay is common. The environmental effects of NICU have also been reported to add to the adversity of prematurity associated neuro-developmental disorder ${ }^{3}$ and scientific evidence of developmental care interventions are inconclusive till date ${ }^{4,5}$. The developmental disorder shall present as a developmental delay, dissociation, deviation and regression ${ }^{6}$. Developmental delay refers to a significant delay in the acquisition of milestones in various developmental domains; deviation refers to atypical acquisition sequence of developmental milestones; dissociation refers to differing rate of development across developmental domains; and
'Selvam Ramachandran, Assistant Professor (Selection Grade) - Physiotherapy, School of Allied Health Sciences, Manipal University, Manipal, Udupi Dist, Karnataka, India. ${ }^{2}$ Dr. Sudip Dutta, Professor and Head Paediatrics, Sikkim Manipal Institute of Medical Sciences, Tadong, Gangtok, East Sikkim, India.

\section{Address for correspondence:}

Selvam Ramachandran, Assistant Professor Physiotherapy, School of Allied Health Sciences, Manipal University, Manipal, Udupi Dist,

Karnataka - 576104, India

E-mail: rs79physio@gmail.com

Tel: +91 9686738297

\section{How to cite}

Ramachandran S, Dutta S. Developmental Screening Tools for Motor Developmental Delay in High Risk Preterm Infants. J Nepal Paediatr Soc 2015;35(2):162-167.

doi: http://dx.doi.org/10.3126/jnps.v35i2.12954

This work is licensed under a Creative Commons Attribution 3.0 License.

\section{(c) (7)}

regression refers to the loss of previously acquired developmental milestones.

The identification of the actual or impending risk of motor developmental disorder are essential to remediate optimal neurodevelopmental outcomes through appropriate early developmental care intervention. Such identification of risk is done through developmental surveillance and screening ${ }^{6,7}$. Dworkin ${ }^{8}$ defined developmental surveillance as "a flexible, continuous process whereby knowledgeable professionals perform 
skilled observations of children during the provision of health care. The components of developmental surveillance include eliciting and attending to parental concerns, obtaining a relevant developmental history, making accurate and informative observations of children, and sharing opinions and concerns with other relevant professionals." Developmental screening ${ }^{9}$ is the administration of a brief standardized tool that aids the identification of children at risk of a developmental disorder. Developmental screening does not result in either a diagnosis or treatment plan but rather identifies areas in which a child's development differs from same-age norms. The American Academy of Pediatrics has issued the policy statement and algorithm on developmental surveillance and screening ${ }^{9}$. The AAP recommends surveillance and screening of all infants to identify established disabilities or risks of delayed development following the AAP algorithm. The algorithm contains recommendations to perform surveillance at all well-child visits and administration of a standardized screening tool at the 9 and 18 month visits and again at either the 24 or 30 month visit. Further it recommends administration of appropriate developmental screening tools for those infants with reported concerns of developmental surveillance.

\section{Characteristics of developmental screening test}

Theoretical framework: The content and constructs of the item set in a developmental screening tool shall be based on neuro-maturational perspective or an ecological perspective ${ }^{10}$. The tool based on biological neuro-maturational perspective assumes that acquisition and performance of motor skills are based on the hierarchical maturation of central nervous system. The tool testing the motor performance based on ecological perspective assumes that the acquisition, performance and maturation of motor skills involve complex interactions of the environmental influences on the developing infant.

Types of test: The screening tests are basically referred as criterion or norm referenced test ${ }^{11}$. The performance score with reference to the attainment of minimum score on the item set tested on specific competencies that marks the pass or fail in the test is termed as criterion referenced test. The performance score when compared with the scores of the normative sample of the similar and larger population is termed as norm referenced test. Care should be taken to draw meaningful inference when using the norm referenced test while comparing the performance score with the normative population as development of motor skills vary amongst diverse social, cultural and ethnic groups.

Test purpose: The clinical examination of preterm infants should include specific and standardized developmental screening tools to discriminate, predict and evaluate motor functions and/or performance. Kirshner B and Guyatt $\mathrm{G}^{12}$ have described a methodological framework for assessing health indices. Based on this framework the developmental screening tool can be classified on basis of test purpose. The discriminative screening tool should be able to distinguish the performance of the subject with or without the function on the specific domain. The norm referenced screening tool will help to discriminate the performance functions of the test group with the normative sample. The predictive screening tool should be able to categorize the test subjects based on the actual or expected performance lags at present or in the future. An evaluative screening tool is used to measure the magnitude of influence of therapeutic interventions on the changes in the performance in a specific domain over a period of time; it helps the health professional to evaluate prognosis on the developmental index as well as the efficacy of early intervention program services.

Test administrator: The administration of test items on a screening tool shall be either a professional with adequate training, expertise and experience or parent / caregiver initiated with little or no training requirement ${ }^{13}$. The test items may be assessed by expert observation or comprehensive elicited examination by the trained health professional.

Age and developmental domains: The appropriateness and utility of the developmental screening tool depends on the age range of subject being tested and the inclusion of constructs of specific developmental domains that are tested ${ }^{14}$.

\section{Challenges of administering standardized developmental screening tool}

The administration of standardized developmental screening tool for motor developmental delay for preterm infants is complex and challenging for the following factors:

Infant factors: The motor development during first year of life follows exponential and non-linear pattern; the unusual and atypical NICU exposure of preterm infants would negatively influence the rapid and critical phase of brain development thus affecting 
the patterns of motor development. The growth and development of preterm infants is atypical of term infants and follows variable motor trajectories ${ }^{11}$. Further the motor development are being influenced and molded by complex psycho-social-cultural factors in the biological milieu of preterm infants.

Screening procedural factors: The use of ageadjusted scores in developmental screening tools remains debatable till date ${ }^{15}$. Some authors recommend that conceptual age (adjusted age) should be taken into account while others maintain that chronological age (no adjustment) should be preferred. Siegel ${ }^{16}$ maintains that "the use of correction will reduce and sometimes remove the apparent difference between the pre-terms and full-terms... it will not necessarily result in the most accurate prediction" of later functioning. The appropriateness of age adjustment depends on the specific domain skills assessed, the degree of prematurity, and the chronological age of the child. The item sets included in the screening tool are clustered within the broad range of items and are placed in correlation to child's age. The ceiling and basal rules are applied to indicate whether the child's performance have reached lower and upper limits. Washington et $\mathrm{al}^{17}$ noted that the clinician's choice of start age item set will increase the chance of under and overestimation of the child's developmental level.

Implementation factors: Countering the test items of the developmental screening tool itself is unnatural for the infant. The test items are administered by the persons (professionals) who are totally unfamiliar with the infant and expecting the infant to remain attentive and carryout adult-directed instructions. Further test items do not engage play-oriented activities during screening. Additional barriers to screening includes lack of confidence / expertise of the health professional, consensus lack on the choice of appropriate screening tool, requirements of tool specific infrastructure and related costs, time constraints, competing clinical demands, cost burden, staffing requirements and logistic issues of working parents in case of longitudinal assessments requiring multiple visits ${ }^{13,18}$.

\section{Use of age-adjusted scores in developmental screening tool}

There are two theoretical viewpoints on the use of age adjustment in preterm infants ${ }^{15}$. From the biological perspective of infant growth and development, the maturation proceeds as a temporal factor since conception. It is rational to infer that there will be lag in one or more developmental domains associated with prematurity; and therefore there is a requirement of age adjustment with a notion that preterm infants will "catch up" with their full term counterparts. From the ecological perspective of infant growth and development, the maturation proceeds as a spatial factor and are influenced by the environmental variables. The parental care, parental stimulation and the parent-child interaction all are said to have influence in growth and development ${ }^{19,20}$.

The preterm birth is not only quantitatively different from full-term birth but also differs qualitatively because of invariable co-morbidities associated with prematurity. Mohr \& Bartelme ${ }^{21}$ introduced the concept of conceptual age also referred to as corrected or adjusted age to overcome the quantitative lag of preterm infants. The performance of preterm infants on a developmental screening tool after age adjustment need not necessarily match with the performance on specific attributes of full term infants. Most of the developmental screening tools for preterm infants encourage use of adjusted age over chronological age.

If preterm infant is being assessed by corrected age, then such infant is being deprived of scores of higher age item set. Hence the standard score may not reflect the appropriate developmental index given the variability in the type of items passed by the preterm infant. The start item set is an important determinant of developmental index on the basis of both chronological and corrected age. The conventional procedure of using the developmental screening tool is that the start item set and the normative group selected should correspond to the chronological age. For preterm infants the items that belong to 'interval' age group (difference in chronological and corrected age) should also be administered. The cumulative use of chronological and corrected age will enable converting the raw score to the standard score in having a meaningful interpretation of achieved developmental index. On one hand, the clinician shall choose the item sets of corrected age and the derived scores shall be compared with the scores of the normative sample of corrected age; on the other, the item sets of chronological age shall be chosen and the derived scores shall be compared with the scores of the normative samples of corrected or chronological age.

In convention and also in several studies, the use of age adjusted developmental screening in clinical practice for at least first two years of life is been recommended. Some researchers also advocate age 
adjusted correction only for the first year of life. It is further stated that "as the child becomes older, this correction factor becomes proportionately smaller compared with total age". Several authors reports the significance of use of age adjusted scores for assessing motor skills in preterm infants for varying periods. Lems et $\mathrm{a}^{22}$ recommends age adjustment for the first six months of the first year while assessing motor skills. Ross ${ }^{23}$ advocated the full age adjustment in assessing motor skills during first year of life as growth and development of motor skills predominates and has a greater impact than on mental skills. Palisano ${ }^{24}$ advocates the use of age adjustment for motor skills until 18 months.

\section{Clinimetric and psychometric standards for effective screening tests}

Developmental screening has become an integral part of quality health care in Developmental Care Interventions (DCI). The health professionals in developmental care of preterm infants should have sound knowledge on the intended purpose of developmental screening tools. The good developmental screening tool should meet the standards of clinimetric and psychometric properties ${ }^{11,13,18,25}$. Such tools should poses varied characteristics such as;

Reliability: It refers to how consistently screening tool identifies children with delays and/or disabilities. It also refers to the consistency of scores and so do the performance of the child with change in screening settings, evaluators and the repeat measures. Instruments should be selected with reliability coefficients greater than. 80 and preferably greater than.90

Validity: It refers to how well a tool measures what it intends to measure. Concurrent validity indicates how well the constructs of the developmental screening tool correlates with the same construct of other screening tool. The construct validity indicates the measurement of item in alignment with a theoretical concept. Criterion validity refers to influence of other variables (criterion).

Utility: It refers to clinical utility of the tool viz. the applicability of the appropriate screening tool specific to the age range and the domains that are being tested.

Specificity and Sensitivity: Specificity refers to the property of tool in correctly identifying the infants developing typically and performing at the expected level of standardized assessment. Sensitivity refers to the ability of the screening tool in detecting small differences in between and within groups of test subjects. $A A P^{9}$ recommends the standard screening tool should have specificity and sensitivity at least in the range of $70-80 \%$ or higher.

\section{Developmental disorder specific screening tools}

The developmental disorders shall include delays in the development of speech and language, fine motor, gross motor, social, and problem-solving skills. Those developmental delays are markers for specific developmental conditions that include cerebral palsy, speech and language disorders, learning disabilities, cognitive disability (mental retardation), autism spectrum disorders and vision or hearing impairment. Globallyvariousdevelopmentalscreening tools arebeing used. The screening tests / tools are either completed by parent or the therapist. The parent completed screening tools include responses to questionnaires based on parental observation of the activity performance. The therapist completed screening tools include specific elicited responses or based on therapist observation of activity performance. The widely used therapist completed multi-domain screening tools include Denver Developmental Screening Test (DDST), Bayley Scale of Infant Development (BSID), Bayley Infant Neurodevelopmental Screener (BINS), Battelle Developmental Inventory Screening Test (BDIST), Milani-Comparetti Development Screening Test (MCDST). The parents completed screening tools include Ages and Stages Questionnaire (ASQ), Kent Inventory of Developmental Skills (KIDS) and Parents' Evaluations of Developmental Status (PEDS) ${ }^{26}$.

\section{Developmental screening tools in Indian context}

\section{Ages and Stages Questionnaire (ASQ)}

Ages and Stages Questionnaire (ASQ), is a set of 19 parent completed questionnaire that are used to evaluate the following developmental domains: communication, gross motor, fine motor, problemsolving, and personal adaptive skills, for children 4 to 60 months old. Domain scores are obtained by the sum of the item scores. Children with ASQ score below the cut off $(\angle 2 S D)$ in any of the domain are taken as screen failed. Juneja $\mathrm{M}$ et $\mathrm{al}^{27}$ reports that $\mathrm{ASQ}$ has strong test characteristics for detecting developmental delay in Indian children and reaffirms the value of ASQ as an effective developmental screening tool. The sensitivity of ASQ is higher in the high risk group, whereas specificity is higher in low risk group. 


\section{Developmental Assessment Scale for Indian Infants (DASII)}

It assesses development in the age range of birth to 30 months and provides a measure of motor and mental development as Motor Developmental Quotient (MoDQ) and Mental Developmental Quotient (MeDQ), respectively. Developmental delay is defined on DASII as DQ score $\leq 70(\leq 2 S D)$ in either the mental or motor scale ${ }^{27,28}$.

\section{Trivandrum Developmental Screening Chart (TDSC)}

The TDSC was designed by selected 17 test items from BSID (Baroda Norms). It was validated both at hospital and community level against the standard Denver Developmental Screening Test. TDSC had a sensitivity of $66.7 \%$ and specificity of $78.8 \%$ which makes it an acceptable simple screening tool even for the community level worker ${ }^{29}$.

Disability Screening Schedule (DSS): It is a broad based onetime screening schedule for all the major disabilities, viz., locomotor, visual, hearing and intellectual in early childhood (0-6 years). DSS has a sensitivity of 0.89 and a specificity of $0.98^{30}$.

\section{Implications for clinical practice}

There is a strong need for primary care provider modulated $^{31}$ multi-disciplinary, community oriented and family centered early intervention developmental care services $^{32}$. The information on the developmental index of preterm infants should be drawn from both the professionally generated developmental screening and with the anecdotal observations of parents / caregivers. The parent completed screening assessment should precede a professionally directed screening as the former one includes observation in a natural environment and specific to socio-cultural practices of family. The appropriate diagnostic tests should be carried out concurrent to developmental screening assessment to make the early intervention services effective ${ }^{33}$. The age-adjusted developmental screening of preterm infants should be done at least in the first year and if there are associated issues of birth weight / co-morbidities, the practice of age-adjustment shall be extended to the second year; thereafter there is no significance of age-adjustment ${ }^{23}$. The longitudinal assessment of at risk infants should follow the AAP algorithm of surveillance during well-child visits and screening at 9, 18 and 24 months ${ }^{9}$. The health professional should choose appropriate screening tool with good clinimetric and psychometric standards while performing the developmental screening tests conforming to the best evidence available ${ }^{34}$.

Funding: Indian Council of Medical Research, IRIS ID: 2008-07900.

\section{References}

1. Wood NS, Marlow N, Costeloe K, Gibson AT, Wilkinson AR. Neurologic and developmental disability after extremely preterm birth. EPICure Study Group. N Engl J Med. 2000; 343(6): 378-84.

2. Hutton JL, Pharoah PO, Cooke RW, Stevenson RC. Differential effects of preterm birth and small gestational age on cognitive and motor development. Arch Dis Child Fetal Neonatal Ed. 1997; 76(2): F75-81.

3. Raghu Raman TS. NICU Environment - A Need for Change. Indian Pediatr. 1997; 34(5): 414-19.

4. Spittle A, Orton J, Anderson P, Boyd R, Doyle LW. Early developmental intervention programmes post-hospital discharge to prevent motor and cognitive impairments in preterm infants. Cochrane Database Syst Rev 2012, Issue 12. Art. No.: CD005495. DOI: 10.1002/14651858. CD005495.pub3.

5. Sizun J, Westrup B, and the ESF Network Coordination Committee. Early developmental care for preterm neonates: a call for more research. Arch Dis Child Fetal Neonatal Ed. 2004; 89(5): F384-8. doi: 10.1136/adc.2003.025114.

6. Poon JK, LaRosa AC, Pai GS. Developmental delay timely identification and assessment. Indian Pediatr. 2010; 47(5): 415-22.

7. Oberklaid F, Efron D. Developmental delay-identification and management. Aust Fam Physician. 2005; 34(9): 739-42.

8. Dworkin PH. Detection of behavioral, developmental, and psychosocial problems in pediatric primary care practice. Curr Opin Pediatr. 1993; 5(5): 531-6.

9. Council on Children with Disabilities, Section on Developmental Behavioral Pediatrics, Bright Futures Steering Committee and Medical Home Initiatives for Children with Special Needs Project Advisory Committee. Identifying Infants and Young Children with Developmental Disorders in the Medical Home: An Algorithm for Developmental Surveillance and Screening. Pediatrics. 2006; 118(1):405-20. 
10. Case-Smith J. Analysis of current motor development theory and recently published infant motor assessments. Infants Young Child. 1996; 9(1): 29-41.

11. Spittle AJ, Doyle LW, Boyd RN. A systematic review of the clinimetric properties of neuro-motor assessments for preterm infants during the first year of life. Dev Med Child Neurol. 2008; 50(4): 254-66.

12. Kirshner B, Guyatt G. A methodological framework for assessing health indices. J Chronic Dis. 1985; 38(1): 27-36.

13. Mackrides PS, Ryherd SJ. Screening for developmental delay. Am Fam Physician. 2011; 84(5): 544-9.

14. Long TM, Tieman B. Review of two recently published measurement tools: the AIMS and the T.I.M.E. Pediat Phys Ther. 1998; 10(2): 62-6.

15. Wilson SL, Cradock MM. Review: Accounting for Prematurity in Developmental Assessment and the Use of Age-Adjusted Scores. J Pediatr Psychol. 2004; 29(8): 641-9.

16. Siegel, L. Correction for prematurity and its consequences for the assessment of the very low birth weight infant. Child Dev. 1983; 54(5): 1176-88.

17. Washington $K$, Scott DT, Johnson KA, Wendel S, \& Hay AE. The Bayley Scales of Infant Development II and Children with Developmental Delays: A clinical perspective. J Dev Behav Pediatr. 1998; 19(5): 3469.

18. Pool JL, Hourcade JJ. Developmental Screening: A Review of Contemporary Practice. Educ Train Autism Dev Disabil. 2011; 46(2): 267-75.

19. Zahr LK. Predictors of development in premature infants from low-income families: African Americans and Hispanics. J Perinatol. 1999; 19(4): 284-9.

20. Badr LK, Bookheimer S, Purdy I, Deeb M. Predictors of neurodevelopmental outcome for preterm infants with brain injury: MRI, medical and environmental factors. Early Hum Dev. 2009; 85(5): 279-84.

21. Mohr GJ and Bartelme P. Mental and physical development of children prematurely born. Am J Dis Child. 1930; 40: 1000-8.

22. Lems W, Hopkins B, Samson JF. Mental and motor development in preterm infants: the issue of corrected age. Early Hum Dev. 1993; 34(1-2): 11323.

23. Ross G, Lawson K. Using the Bayley-II: unresolved issues in assessing the development of prematurely born children. J Dev Behav Pediatr. 1997; 18(2): 109-11; discussion 112-3.

24. Palisano RJ. Use of Chronological and Adjusted Ages to compare Motor Development of Healthy Preterm and Full-term Infants. Dev Med Child Neurol. 1986; 28(2): 180-7.

25. Macy M. The evidence behind developmental screening instruments. Infants Young Child. 2012; 25(1): 19-61.

26. Ringwalt S. Developmental screening and assessment instruments with an emphasis on social and emotional development for young children aged birth through five. Chapel Hill: The University of North Carolina, FPG Child Development Institute, National Early Childhood Technical Assistance Center; 2008.

27. Juneja $M$, Mohanty $M$, Jain R, Ramji S. Ages and Stages Questionnaire as a screening tool for developmental delay in Indian children. Indian Pediatr. 2012; 49(6): 457-61.

28. Misra N, Pathak P. Developmental assessment scales for Indian Infants (DASII): Manual. Baroda, MS University of Baroda. 1996.

29. Nair MK1, Nair GS, George B, Suma N, Neethu C, Leena ML, Russell PS. Development and Validation of Trivandrum Development Screening Chart for Children Aged 0-6 years [TDSC (0-6)]. Indian J Pediatr. 2013; 80(Suppl 2): S248-255.

30. Chopra G, Verma IC, Seetharaman P. Development and assessment of a screening test for detecting childhood disabilities. Indian J Pediatr. 1999; 66(3): 331-35.

31. Aly Z, Taj F, Ibrahim S. Missed opportunities in surveillance and screening systems to detect developmental delay: A developing country perspective. Brain Dev. 2010; 32(2): 90-7.

32. American Academy of Pediatrics Council on Children with Disabilities, Duby JC. Role of the Medical Home in Family-Centered Early Intervention Services. Pediatrics. 2007; 120(5): 1153-8.

33. Noritz GH, Murphy NA and Neuromotor Screening Expert Panel, American Academy of Pediatrics. Motor Delays: Early Identification and Evaluation. Pediatrics. 2013; 131(6): e2016-27.

34. US National Institutes of Health. Translating Evidence Based Developmental Screening Into Pediatric Primary Care (TEDS). [Internet]. 2009 [updated 2011, Nov 8; cited 2015, Dec 14]. Available from: https://clinicaltrials.gov/ct2/ show/NCT00844246 